\title{
Spectral and coherent properties of partially polarized pulsed electromagnetic beams upon turbulent atmosphere propagation for different source conditions
}

\author{
Yan $\mathrm{Li}^{1} \cdot$ Ming Gao ${ }^{1}$ (1) \\ Received: 24 August 2019 / Accepted: 9 January 2020 / Published online: 7 February 2020 \\ (c) The Author(s) 2020
}

\begin{abstract}
The spectral and coherent properties of partially polarized pulsed electromagnetic beams propagating in a horizontal homogeneous and isotropic turbulent atmosphere channel for different source conditions are investigated in detail. Based on the extended Huygens-Fresnel principle and von Karman power spectrum, the analytical expressions for the elements of the two-frequency cross spectral density matrix, the average spectral intensity, the beam width, and the spectral degree of electromagnetic coherence are derived in turn. Finally, with the help of numerical calculations, the effects of different source conditions on the changes in the beam width, spectral and coherent properties of partially polarized pulsed Gaussian Schellmodel electromagnetic beams are studied. Some theoretical results in this paper will be useful for applications involving polarized pulsed electromagnetic beams propagating through atmospheric turbulence.
\end{abstract}

\section{Introduction}

In the past decades, with the rapid development of femtosecond pulse laser technology, ultrashort pulse beam has aroused great interests of scholars at home and abroad [1-6]. For an optical pulse, it is commonly assumed that both its spatial and spectral components are completely coherent. However, such assumption does not stand in practice, i.e., the spatial and spectral distributions of optical pulses are usually partially coherent, which represent a class of partially coherent non-stationary wave field [7, 8]. In 2002, a scalar model of a partially coherent plane-wave pulse with a Gaussian spectrum and a Gaussian distribution of correlations between different frequency components, i.e., the Gaussian Schell-model pulse (GSMP) was introduced in the frequency domain by Paakkonen et al. [9] and later in the temporal domain by Lin et al. [10]. The so-called Gaussian Schell-model beam, is a convenient mathematical model for wave fields which are spatially partially coherent, which helps to understand the role of partial spatial coherence in physical radiometry

Ming Gao

minggao1964@163.com

1 School of Optoelectronic Engineering, Xi'an Technological University, No. 2 Xuefu Road, Weiyang District, Xi'an 710021, China
[9]. Therefore, using analogy to this model, the spectral and temporal components of the Gaussian Schell-model pulse is obtained [11]. Since then, generation, propagation, and coherent-mode representation of such a partially coherent pulse beam have been studied in detail [12-20]. It is noted that the above researches are all based on scalar fields.

On the other hand, coherence and polarization are natural physical characteristics of light [21]. In 2003, Wolf proposed the unified theory of coherence and polarization of stochastic electromagnetic beams [22], i.e., vector light field. Henceforth, there has been numerous interests in studying the spectral, coherent, and polarization properties, etc., of stochastic electromagnetic (pulse) beams on propagation through different kinds of random mediums, such as a gradient-index fiber [23], an optical system [24, 25], dispersive media [26], free space [27-29], turbulent atmosphere [30], and oceanic turbulence [31]. Nevertheless, literature mentioned above have been restricted to the electromagnetic pulse beams by assuming that the electric vector components in the $x$ and $y$ directions are uncorrelated at the source plane. Recently, Voipio et al. presented the coherent-mode decomposition for pulsed electromagnetic beam fields and introduced the overall degree of coherence as a measure of the average temporal or spectral and spatial coherence of the beam [32]. However, to the best of our knowledge, rarely the references are concerned with the context of propagation properties of the partially polarized, partially spatially and 
spectrally coherent pulsed electromagnetic fields through turbulent atmosphere.

In this work, we take the partially polarized pulsed electromagnetic Gaussian Schell-model (PEGSM) beams as a typical example, which are also called as partially polarized electromagnetic Gaussian Schell-model pulse (EGSMP) beams. The paper is organized as follows. In Sect. 2, the beam model in source plane is established and analytical expressions of the elements of the two-frequency cross spectral density matrix and the average spectral intensity, the beam width and the spectral degree of electromagnetic coherence in the observation plane are derived in turn. Simulation results are presented and discussed in Sect. 3. The influence of different source conditions and turbulent atmosphere on the normalized spectral intensity, beam width in the $x$ and $y$ directions and two-frequency, two points spectral degree of electromagnetic coherence are emphasized. Finally, the main results obtained in this paper are summarized in Sect. 4.

\section{Theoretical formulation}

We consider optical wave field propagation from the source plane $z=0$ to the observation plane $z>0$ where a horizontal homogeneous and isotropic turbulent atmosphere channel exists. In space-time domain, the elements of the polarization-coherence function matrix of the partially polarized PEGSM beams in $z=0$ are given by the expressions:

$\Gamma^{(0)}\left(\mathbf{r}_{1}, \mathbf{r}_{2}, 0, t_{1}, t_{2}\right)=\left[\begin{array}{l}\Gamma_{x x}^{(0)}\left(\mathbf{r}_{1}, \mathbf{r}_{2}, 0, t_{1}, t_{2}\right) \Gamma_{x y}^{(0)}\left(\mathbf{r}_{1}, \mathbf{r}_{2}, 0, t_{1}, t_{2}\right) \\ \Gamma_{y x}^{(0)}\left(\mathbf{r}_{1}, \mathbf{r}_{2}, 0, t_{1}, t_{2}\right) \Gamma_{y y}^{(0)}\left(\mathbf{r}_{1}, \mathbf{r}_{2}, 0, t_{1}, t_{2}\right)\end{array}\right]$,

with

$$
\begin{aligned}
\Gamma_{i j}^{(0)}\left(\mathbf{r}_{1}, \mathbf{r}_{2}, 0, t_{1}, t_{2}\right)= & A_{i} A_{j} B_{i j} \exp \left[-\frac{\mathbf{r}_{1}^{2}}{4 \sigma_{i}^{2}}-\frac{\mathbf{r}_{2}^{2}}{4 \sigma_{j}^{2}}-\frac{\left(\mathbf{r}_{1}-\mathbf{r}_{2}\right)^{2}}{2 \delta_{i j}^{2}}\right] \\
& \exp \left[-\frac{t_{1}^{2}+t_{2}^{2}}{2 T_{0}^{2}}-\frac{\left(t_{1}-t_{2}\right)^{2}}{2 T_{c i j}^{2}}-i \omega_{0}\left(t_{1}-t_{2}\right)\right] .
\end{aligned}
$$

Here the variances $\sigma_{i}, \delta_{i j}$ are independent of position but may depend on frequency. $\sigma_{i}(i=x, y)$ is initial beam waist width in $i$ - direction. $A_{i}$ denotes the amplitude of $i$-direction. In addition, the parameters $B_{i j}$ have the following properties [27, 33]: $B_{i j} \equiv 1$ when $i=j,\left|B_{i j}\right| \leq 1$ when $i \neq j$, and $B_{x y}=B^{*}{ }_{y x}=$ $\left|B_{x y}\right| \operatorname{lexp}(i \theta), \theta$ is the phase difference, $\theta \neq k \pi(k=1,2,3, \ldots)$. $T_{0}$ is the pulse duration, $\omega_{0}$ is center frequency. $\delta_{i j}$ and $T_{c i j}$ are the spatial and temporal coherent width of the beam, respectively. The beam parameters $\sigma_{x}, \sigma_{y}, \delta_{i}$, and $T_{c i j}$, which satisfy the following intrinsic constraints $[33,34]$ :

$$
\left\{\begin{array}{l}
\frac{1}{4 \sigma_{i}^{2}}+\frac{1}{\delta_{i i}^{2}} \leq \frac{2 \pi^{2}}{\lambda^{2}},(i=x, y), \\
\max \left\{\delta_{x x}, \delta_{y y}\right\} \leq \delta_{x y} \leq \min \left\{\frac{\delta_{x x}}{\left|B_{x y}\right|}, \frac{\delta_{y y}}{\left|B_{x y}\right|}\right\}, \\
\max \left\{T_{c x x}, T_{c y y}\right\} \leq T_{c x y} \leq \min \left\{\frac{T_{c x x}}{\left|B_{x y}\right|}, \frac{T_{c y y}}{\left|B_{x y}\right|}\right\} .
\end{array}\right.
$$

In the following numerical examples, the values of $\sigma_{i}, \delta_{i j}$, and $T_{c i j}$, are chosen to satisfy Eq. (3).

Using the Fourier-transform [30], the element of the twofrequency cross spectral density matrix can be expressed as:

$\mathbf{W}\left(\mathbf{r}_{1}, \mathbf{r}_{2}, 0, \omega_{1}, \omega_{2}\right)=\iint \Gamma\left(\mathbf{r}_{1}, \mathbf{r}_{2}, 0, t_{1}, t_{2}\right) \exp \left[i\left(\omega_{1} t_{1}-\omega_{2} t_{2}\right)\right] d t_{1} d t_{2}$.

Substituting Eqs. (1), (2) into Eq. (4), we can get

$$
\begin{aligned}
W_{i j}\left(\mathbf{r}_{1}, \mathbf{r}_{2}, 0, \omega_{1}, \omega_{2}\right)= & \frac{A_{i} A_{j} B_{i j} T_{0}}{2 \pi \Omega_{0 i j}} \\
& \exp \left[-\frac{\mathbf{r}_{1}^{2}}{4 \sigma_{i}^{2}}-\frac{\mathbf{r}_{2}^{2}}{4 \sigma_{j}^{2}}-\frac{\left(\mathbf{r}_{1}-\mathbf{r}_{2}\right)^{2}}{2 \delta_{i j}^{2}}\right] \\
& \exp \left[-\mathrm{T}_{i j}\left(\omega_{1}, \omega_{2}\right)\right],
\end{aligned}
$$

where

$\mathrm{T}_{i j}\left(\omega_{1}, \omega_{2}\right)=\frac{\left(\omega_{1}-\omega_{0}\right)^{2}+\left(\omega_{2}-\omega_{0}\right)^{2}}{2 \Omega_{0 i j}^{2}}+\frac{\left(\omega_{1}-\omega_{2}\right)^{2}}{2 \Omega_{c i j}^{2}}$,

where, $\Omega_{0 i j}=\sqrt{1 / T_{0}^{2}+2 / T_{c i j}^{2}}$ and $\Omega_{c i j}=T_{c i j} \Omega_{0 i j} / T_{0}$ describe the spectral width and the spectral coherent width of the beam, respectively.

When the partially polarized PEGSM beams propagate through a horizontal homogeneous and isotropic atmospheric turbulence from the source plane to the observation plane $(z>0)$ along the $z$-axis, the position coordinates of any two points on the $z$ plane are expressed by $\boldsymbol{\rho}_{1}=\left(x_{1}, y_{1}\right)$ and $\boldsymbol{\rho}_{2}=\left(x_{2}, y_{2}\right)$, respectively. According to the extended Huygens-Fresnel principle, the elements of the two-frequency cross spectral density matrix can be expressed as [22]:

$$
\begin{aligned}
W_{i j}\left(\boldsymbol{\rho}_{1}, \boldsymbol{\rho}_{2}, z ; \omega_{1}, \omega_{2}\right)= & \frac{k_{1} k_{2}}{4 z^{2} \pi^{2}} \exp \left[-i\left(k_{1}-k_{2}\right) z\right] \\
& \int_{-\infty}^{\infty} \iiint d^{2} \mathbf{r}_{1} d^{2} \mathbf{r}_{2} W_{i j}\left(\mathbf{r}_{1}, \mathbf{r}_{2}, 0 ; \omega_{1}, \omega_{2}\right) \\
& \times \exp \left\{-\frac{i}{2 c z}\left[\omega_{1}\left(\boldsymbol{\rho}_{1}-\mathbf{r}_{1}\right)^{2}-\omega_{2}\left(\boldsymbol{\rho}_{2}-\mathbf{r}_{2}\right)^{2}\right]\right\} \\
& \times C_{\psi}\left(\mathbf{r}_{1}, \boldsymbol{\rho}_{1}, \mathbf{r}_{2}, \boldsymbol{\rho}_{2}, z, k_{1}, k_{2}\right),
\end{aligned}
$$

where $k=\omega / c$, is the wave number, and $c=3 \times 10^{8} \mathrm{~m} / \mathrm{s}$. $C_{\psi}$ $\left(\mathbf{r}_{1}, \boldsymbol{\rho}_{1}, \mathbf{r}_{2}, \boldsymbol{\rho}_{2}, z, k_{1}, k_{2}\right)$ denotes the random phase fluctuation 
caused by turbulence. This paper is based on the second order statistics (i.e. mutual coherence function or cross spectral density); therefore, we will assume that the extended Huygen's Fresnel approach is valid in all regimes of atmospheric turbulence with respect to propagation properties [35]. Paralleling the pulse wave analysis in Ref. [35], the complex phase fluctuation is given by:

$$
\begin{aligned}
& C_{\psi}\left(\mathbf{r}_{1}, \boldsymbol{\rho}_{1}, \mathbf{r}_{2}, \rho_{2}, z, k_{1}, k_{2}\right)=\left\langle\exp \left[\psi\left(\mathbf{r}_{1}, \boldsymbol{\rho}_{1}, z, k_{1}\right)+\psi^{*}\left(\mathbf{r}_{2}, \boldsymbol{\rho}_{2}, z, k_{2}\right)\right]\right\rangle \\
& \cong \Gamma_{2} \exp \left[-\frac{1}{2} D_{\mathrm{sp}}\left(\mathbf{r}_{1}-\mathbf{r}_{2}, \rho_{1}-\boldsymbol{\rho}_{2}\right)\right] \\
& \Gamma_{2}=\exp \left[-2 \pi^{2}\left(k_{1}-k_{2}\right)^{2} z \int_{0}^{\infty} \kappa \Phi_{n}(\kappa) d \kappa\right], D_{\mathrm{sp}}(Q) \\
&=8 \pi^{2} k_{1} k_{2} z \int_{0}^{1} \int_{0}^{\infty} \kappa \Phi_{n}(\kappa)\left(1-J_{0}(\kappa \xi|Q|)\right)
\end{aligned}
$$

where $J_{0}$ is a Bessel function and $\Phi_{n}(\kappa)$ is the refractive index power spectrum. Dsp $(Q)$ is the spherical wave structure function. In our analysis, we use the von Karman spectrum given by:

$\Phi_{n}(\kappa)=0.033 C_{n}^{2} \frac{\exp \left(-\kappa^{2} / \kappa_{m}^{2}\right)}{\left(\kappa^{2}+\kappa_{0}^{2}\right)^{11 / 6}}$,

where $\kappa_{m}=5.92 / l_{0}, \kappa_{0}=1 / L_{0}, L_{0}$ and $l_{0}$ are the outer and inner scales of turbulence, respectively; $\kappa$ is the spatial radial frequency, and $C_{n}^{2}$ is the structure parameter.

The spherical wave structure function $\operatorname{Dsp}(Q)$ and $\Gamma_{2}$ in Eq. (9) based on the von Karman spectrum have been shown to be approximated by [35]:

$D_{\text {sp }}(Q) \approx 2\left(\frac{Q}{\rho_{0}}\right)^{2}, \Gamma_{2}=\exp \left(-0.391 C_{n}^{2} k_{d}^{2} z L_{0}^{5 / 3}\right)$,

respectively. In Eq. (11), $\rho_{0}=\left(0.55 C_{n}^{2} k_{c}^{2} l_{0}^{-1 / 3} z\right)^{-1 / 2}$ is the coherence length of a spherical wave propagating in the horizontal path of turbulent medium, $k_{c}=\left(k_{1}+k_{2}\right) / 2$, $k_{d}=k_{1}-k_{2}$.

In Ref. [35], $Q$ only includes the points on-axis and ignores the points off-axis, therefore, it can be extended in this paper to include all points in the observation plane. i.e.,

$D_{\mathrm{sp}}(Q) \cong 2 \frac{\left(\mathbf{r}_{1}-\mathbf{r}_{2}\right)^{2}+\left(\mathbf{r}_{1}-\mathbf{r}_{2}\right)\left(\rho_{1}-\rho_{2}\right)+\left(\rho_{1}-\rho_{2}\right)^{2}}{\rho_{0}^{2}}$.

Substituting Eqs. (5), (6) and Eqs. (8)-(12) into Eq. (7) and calculating the related integral we obtain:

$$
\begin{aligned}
W_{i j}\left(\rho_{1}, \rho_{2}, z, \omega_{1}, \omega_{2}\right)= & \frac{\omega_{1} \omega_{2} W_{0 i j} \Gamma_{2} \gamma_{i j}^{4}}{c^{2} z^{2}\left(4 \alpha_{1 i j} \alpha_{2 i j} \gamma_{i j}^{4}-1\right)} \\
& \exp \left[\frac{i}{2 c z}\left(\omega_{2} \rho_{2}^{2}-\omega_{1} \rho_{1}^{2}\right)-\frac{\left(\rho_{1}-\rho_{2}\right)^{2}}{\rho_{0}^{2}}\right] \\
& \exp \left[\frac{\gamma_{i j}^{4}\left(p^{2} \alpha_{2 i j}+q^{2} \alpha_{1 i j}\right)-p q \gamma_{i j}^{2}}{4 \alpha_{1 i j} \alpha_{2 i j} \gamma_{i j}^{4}-1}\right] \\
& \times \exp \left[\frac{-i\left(\omega_{1}-\omega_{2}\right) z}{c}\right. \\
& \left.-\frac{\left(\omega_{1}-\omega_{0}\right)^{2}+\left(\omega_{2}-\omega_{0}\right)^{2}}{2 \Omega_{0 i j}^{2}}-\frac{\left(\omega_{1}-\omega_{2}\right)^{2}}{2 \Omega_{c i j}^{2}}\right],
\end{aligned}
$$

with

$W_{0 i j}=\frac{A_{i} A_{j} B_{i j} T_{0}}{2 \pi \Omega_{0 i j}}$,

$\alpha_{1 i j}=\frac{1}{4 \sigma_{i}^{2}}+\frac{1}{2 \delta_{i j}^{2}}+\frac{1}{\rho_{0}^{2}}+\frac{i \omega_{1}}{2 c z}$

$\alpha_{2 i j}=\frac{1}{4 \sigma_{j}^{2}}+\frac{1}{2 \delta_{i j}^{2}}+\frac{1}{\rho_{0}^{2}}-\frac{i \omega_{2}}{2 c z}$,

$\frac{1}{\gamma_{i j}^{2}}=\frac{1}{\delta_{i j}^{2}}+\frac{2}{\rho_{0}^{2}}, p=\frac{i \omega_{1}}{c z} \rho_{1}-\frac{\left(\rho_{1}-\rho_{2}\right)}{\rho_{0}^{2}}$,

$q=\frac{i \omega_{2}}{c z} \rho_{2}-\frac{\left(\rho_{1}-\rho_{2}\right)}{\rho_{0}^{2}}$.

\subsection{Average spectral intensity}

The average spectral intensity at the point $(\rho, z, \omega)$ was given by the formula [27]:

$$
\begin{aligned}
S(\rho, z, \omega) & =\operatorname{Tr}\left(W_{i j}(\rho, \rho, z, \omega, \omega)\right) \\
& =\frac{T_{0}}{2 \pi}\left\{\frac{1}{\Omega_{0 x x}} \frac{A_{x}^{2}}{\Delta_{x x}} \exp \left[-\frac{\rho^{2}}{2 \sigma_{x}^{2} \Delta_{x x}}\right] \exp \left[-\frac{\left(\omega-\omega_{0}\right)^{2}}{\Omega_{0 x x}^{2}}\right]\right. \\
& \left.+\frac{1}{\Omega_{0 y y}} \frac{A_{y}^{2}}{\Delta_{y y}} \exp \left[-\frac{\rho^{2}}{2 \sigma_{y}^{2} \Delta_{y y}}\right] \exp \left[-\frac{\left(\omega-\omega_{0}\right)^{2}}{\Omega_{0 y y}^{2}}\right]\right\},
\end{aligned}
$$

where

$\Delta_{x x}=1+\frac{c^{2}}{2 \sigma_{x}^{2} \omega^{2}}\left(\frac{1}{2 \sigma_{x}^{2}}+\frac{2}{\delta_{x x}^{2}}\right) z^{2}+1.1 C_{n}^{2} \sigma_{x}^{-2} l_{0}^{-1 / 3} z^{3}$,

$\Delta_{y y}=1+\frac{c^{2}}{2 \sigma_{y}^{2} \omega^{2}}\left(\frac{1}{2 \sigma_{y}^{2}}+\frac{2}{\delta_{y y}^{2}}\right) z^{2}+1.1 C_{n}^{2} \sigma_{y}^{-2} l_{0}^{-1 / 3} z^{3}$.

Equation (16) is similar to Eq. (15) in [30], which is employing the Kolmogorov spectrum model. By setting $\boldsymbol{\rho}=(0,0)$, we can get on-axis average spectral intensity: 


$$
\begin{aligned}
S(0, z, \omega)= & \frac{T_{0}}{2 \pi \Omega_{0 x x}} \frac{A_{x}^{2}}{\Delta_{x x}} \exp \left[-\frac{\left(\omega-\omega_{0}\right)^{2}}{\Omega_{0 x x}^{2}}\right] \\
& +\frac{T_{0}}{2 \pi \Omega_{0 y y}} \frac{A_{y}^{2}}{\Delta_{y y}} \exp \left[-\frac{\left(\omega-\omega_{0}\right)^{2}}{\Omega_{0 y y}^{2}}\right] .
\end{aligned}
$$

It is helpful to define the normalized spectral intensity as:

$S^{\prime}(\rho, z, \omega)=S(\rho, z, \omega) / S_{\max }(\rho, z, \omega)$.

The average spectral intensity for the partially polarized PEGSM beam is determined in Eq. (15), and the change in the spectral property on propagation in a homogeneous and isotropic turbulent atmosphere is studied by choosing different source conditions parameters $\sigma_{i}, \delta_{i j}$ and $T_{c i j}$, refractive index structure constant $C_{n}^{2}$, and turbulence inner scale $l_{0}$.

\subsection{Beam width}

Let us study how the source conditions and turbulence affect the spot size of the beam. We define the width $W$ of the beam spot such that [36]

$\left\{S\left(z, \boldsymbol{\rho}_{c}=W, \boldsymbol{\rho}_{d}=0\right) / S\left(z, \boldsymbol{\rho}_{c}=\boldsymbol{\rho}_{d}=0\right)\right\}=\exp (-2)$,

where $\boldsymbol{\rho}_{c}=\left(\boldsymbol{\rho}_{1}+\boldsymbol{\rho}_{2}\right) / 2, \boldsymbol{\rho}_{d}=\boldsymbol{\rho}_{1}-\boldsymbol{\rho}_{2}$.

Substituting Eqs. (15), (16) into Eq. (19), we obtain the following equation for the beam size $W_{x}(z, \omega)$ and $W_{y}(z, \omega)$ can be written as:

$$
\begin{aligned}
W_{x}(z, \omega) & =\sigma_{x} \sqrt{\Delta_{x x}} \\
& =\sqrt{\sigma_{x}^{2}\left[1+\frac{c^{2}}{2 \omega^{2} \sigma_{x}^{2}}\left(\frac{1}{2 \sigma_{x}^{2}}+\frac{2}{\delta_{x x}^{2}}\right) z^{2}\right]+1.1 C_{n}^{2} l_{0}^{-1 / 3} z^{3}} \\
& =\sqrt{\sigma_{x}^{2}+\frac{\sigma_{x}^{2} z^{2}}{16 Z_{R x}^{2}}+\frac{c \sigma_{x}^{2} z^{2}}{2 \omega Z_{R x} \delta_{x x}^{2}}+1.1 C_{n}^{2} l_{0}^{-1 / 3} z^{3},} \\
W_{y}(z, \omega) & =\sigma_{y} \sqrt{\Delta_{y y}} \\
& =\sqrt{\sigma_{y}^{2}\left[1+\frac{c^{2}}{2 \omega^{2} \sigma_{y}^{2}}\left(\frac{1}{2 \sigma_{y}^{2}}+\frac{2}{\delta_{y y}^{2}}\right) z^{2}\right]+1.1 C_{n}^{2} l_{0}^{-1 / 3} z^{3}} \\
& =\sqrt{\sigma_{y}^{2}+\frac{\sigma_{y}^{2} z^{2}}{16 Z_{R y}^{2}}+\frac{c \sigma_{y}^{2} z^{2}}{2 \omega Z_{R y} \delta_{y y}^{2}}+1.1 C_{n}^{2} l_{0}^{-1 / 3} z^{3},}
\end{aligned}
$$

The term $Z_{R i}=k \sigma_{i}^{2} / 2$ denotes the Rayleigh distance. It can be seen from Eqs. (20) and (21) that the second and third terms in the square root stand for diffraction of the beam on free-space propagation induced by $\sigma_{i}$ and $\delta_{i i}(i=x$, $y$ ), respectively. Diffraction in the two terms is proportional to the square of the propagation distance $z$. The fourth term stands for the extra diffraction induced by turbulence and proportional to the cube of the propagation distance $z$, also proportional to the turbulence structure parameter $C_{n}{ }^{2}$, and inversely proportional to the one third power of the turbulence inner scale $l_{0}$.

We noticed that in free space $\left(C_{n}{ }^{2}=0\right)$ the beam size remains close to $\sigma_{i}$ as long as $z<<Z_{R i}$. The beam begins to diverge rapidly in the far-field region $z>Z_{R i}$. The presence of the turbulence medium causes the beam to diverge further.

\subsection{Spectral degree of electromagnetic coherence}

Correlations between different electric-field frequency components are characterized by the spectral degree of electromagnetic coherence $\mu_{\mathrm{EM}}\left(\boldsymbol{\rho}_{1}, \boldsymbol{\rho}_{2}, z, \omega_{1}, \omega_{2}\right)$ originally introduced for stationary, spatially partially coherent fields [32], but which can readily be extended to spatially and spectrally partially coherent fields as:

$\mu_{\mathrm{EM}}^{2}\left(\rho_{1}, \boldsymbol{\rho}_{2}, z, \omega_{1}, \omega_{2}\right)=\frac{\left\|\mathbf{W}\left(\rho_{1}, \rho_{2}, z, \omega_{1}, \omega_{2}\right)\right\|_{F}^{2}}{S\left(\rho_{1}, z, \omega_{1}\right) S\left(\rho_{2}, z, \omega_{2}\right)}$,

where $\|\cdot\|_{F}$ is the Frobenius norm.

From Eq. (22), we can get three models for spectral degree of electromagnetic coherence, i.e., (1) two-frequency, single- point spectral degree of electromagnetic coherence $\mu_{\mathrm{EM}}\left(\boldsymbol{\rho}, \boldsymbol{\rho}, z, \omega_{1}, \omega_{2}\right) ;(2)$ single-frequency, two-point spectral degree of electromagnetic coherence $\mu_{\mathrm{EM}}\left(\boldsymbol{\rho}_{1}, \boldsymbol{\rho}_{2}, z, \omega\right.$, $\omega$ ); and (3) two-frequency, two-point spectral degree of electromagnetic coherence $\mu_{\mathrm{EM}}\left(\boldsymbol{\rho}_{1}, \boldsymbol{\rho}_{2}, z, \omega_{1}, \omega_{2}\right)$. For the sake of simplicity, in this work, we take the two-frequency, two-point spectral degree of electromagnetic coherence as a typical example, which can be written as:

$\mu_{\mathrm{EM}}\left(0, d, z, \omega_{1}, \omega_{2}\right)=\sqrt{\frac{\left\|\mathbf{W}\left(0, d, z, \omega_{1}, \omega_{2}\right)\right\|_{F}^{2}}{S\left(0, z, \omega_{1}\right) S\left(d, z, \omega_{2}\right)}}$,

where we set $\rho_{1}=(0,0)$ and $\rho_{2}=(d, 0)$. When $d=0$, Eq. (23) becomes the two-frequency, single-point spectral degree of electromagnetic coherence $\mu_{\mathrm{EM}}\left(0,0, z, \omega_{1}, \omega_{2}\right)$.

\section{Numerical results and discussion}

To evaluate the preceding analysis, we show the detailed variations of propagation properties parameters in a horizontal homogeneous and isotropic turbulent atmosphere channel. Here, the different conditions' source parameters, i.e., isotropic source, semi-isotropic source and the anisotropic source [37], are adopted as:

(1) Isotropic source: $\sigma_{x}=\sigma_{y}=2 \mathrm{~cm}, \delta_{x x}=\delta_{y y}=2 \mathrm{~mm}$, $T_{c x x}=T_{c y y}=30 \mathrm{fs}$;

(2) Semi-isotropic source: $\sigma_{x}=1 \mathrm{~cm}, \sigma_{y}=2 \mathrm{~cm}$, $\delta_{x x}=\delta_{y y}=2 \mathrm{~mm}, T_{c x x}=T_{c y y}=30 \mathrm{fs}$; 


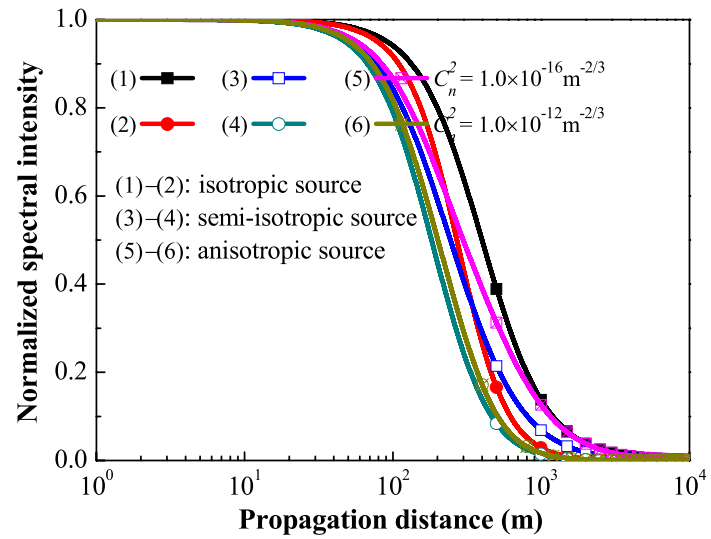

Fig. 1 Evolution of on-axis normalized spectral intensity with $z$ for various values of $C_{n}^{2}$ and different source conditions

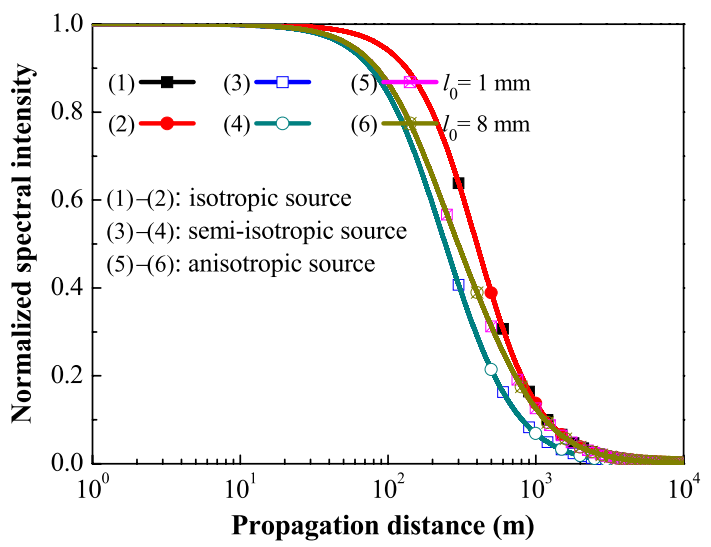

Fig. 2 Evolution of on-axis normalized spectral intensity with $z$ for various values of $l_{0}$ and different source conditions

(3) Anisotropic source: $\sigma_{x}=1 \mathrm{~cm}, \sigma_{y}=2 \mathrm{~cm}, \delta_{x x}=2 \mathrm{~mm}$, $\delta_{y y}=3 \mathrm{~mm}, T_{c x x}=20 \mathrm{fs}, T_{c y y}=30 \mathrm{fs}$.

The other parameters are choosen to be: $\omega_{0}=2.98 \times 10^{15} \mathrm{rad} / \mathrm{s} \quad(\lambda=632.8 \mathrm{~nm})$, $\omega=\omega_{1}=3.0 \times 10^{15} \mathrm{rad} / \mathrm{s}, \omega_{2}=2.96 \times 10^{15} \mathrm{rad} / \mathrm{s}, A_{x}=1.5$, $A_{y}=1, T_{0}=50 \mathrm{fs}, B_{x y}=0.3 \exp (i \pi / 3), \delta_{x y}=4 \mathrm{~mm}, T_{c x y}=$ $50 \mathrm{fs}, C_{n}^{2}=10^{-14} \mathrm{~m}^{-2 / 3}, l_{0}=1 \mathrm{~mm}, L_{0}=10 \mathrm{~m}$.

We employ the above parameters as examples for a theoretical analysis; other values can also be chosen depending on the propagation scenario. The above parameters are measured in a turbulent channel, consistent with physically realizable conditions. The results are shown in Figs. 1, 2, 3, $4,5,6,7,8,9,10,11$.

Now we study the evolution of the on-axis normalized spectral intensity of a partially polarized PEGSM beam in a horizontal homogeneous and isotropic turbulent atmosphere for different source conditions. By applying Eqs. (16)-(18), we calculate in Fig. 1 the on-axis normalized spectral intensity $S^{\prime}(0, z, \omega)$ versus the propagation distance $z$ for different source conditions and various values of refractive index structure constant $C_{n}{ }^{2}$. As can be seen, values of $S^{\prime}(0, z, \omega)$ near the source plane $(z \leq 50 \mathrm{~m})$ is unchanged for different source conditions and different values of $C_{n}{ }^{2}$. For the case of $100 \mathrm{~m} \leq z \leq 1000 \mathrm{~m}$, the values of $S^{\prime}(0, z, \omega)$ decrease rapidly with the increase of $z$; fixing $z$ and $C_{n}^{2}$, value of $S^{\prime}(0, z, \omega)$ for isotropic source is the largest and semi-isotropic source is the smallest. The smaller $C_{n}^{2}$ is, the greater the difference between the three sources will become. When $C_{n}{ }^{2}$ is larger, the curves of $S^{\prime}$ $(0, z, \omega)$ are almost overlapped for the case of the latter two sources.

In Fig. 2, the $S^{\prime}(0, z, \omega)$ is plotted for several values of turbulence inner scale $l_{0}$. As can be seen, the evolution of $S^{\prime}(0, z, \omega)$ is consistent with Fig. 1 . For different $l_{0}$, the value of $S^{\prime}(0, z, \omega)$ is almost unchanged. In other words, the turbulence inner scale hardly affects the $S^{\prime}(0, z, \omega)$. On the other hand, the source parameters have great effect on the $S^{\prime}$ $(0, z, \omega)$, for the case of $100 \mathrm{~m} \leq z \leq 1000 \mathrm{~m}$.

By applying Eqs. (15) and (18), we calculate in Fig. 3 the normalized 3D-spectral intensity distribution $S(x, y, z$, $\omega) / S_{\max }(x, y, z, \omega)$ of a partially polarized PEGSM beam at several propagation distances in a horizontal homogeneous and isotropic turbulent atmosphere for different source conditions (a) isotropic source, (b) semi-isotropic source, and (c) anisotropic source. To learn about evolution of the average spectral intensity of a partially polarized PEGSM beam on propagation, we also calculate in Fig. 4 the cross line $(y=0)$ of the normalized average spectral intensity distribution $S(x, 0, z, \omega) / S_{\max }(x, 0, z, \omega)$ of a partially polarized PEGSM beam at several propagation distances during propagation for different source conditions. One sees from Figs. 3 and 4 that in the far field region, the partially polarized partially coherent PEGSM beam propagating through the turbulent atmosphere can keep its initial beam profile almost unchanged. Moreover, the beam shapes of the three sources are similar. The beam profiles of both the isotropic and the semi-isotropic source almost coincide in the far field, and the center width of spectral intensity distribution in the case of the anisotropic source is always smaller than the other two cases.

The relative spectral shift $\delta \omega / \omega_{0}$ is defined as $\delta \omega /$ $\omega_{0}=\left(\omega_{\max }-\omega_{0}\right) / \omega_{0}$, where $\omega_{\max }$ denotes the frequency at which the spectrum takes the maximum value. The normalized spectral intensity $S^{\prime}(\rho, z, \omega)$ versus the relative spectral shift $\delta \omega / \omega_{0}$ at the observations (a) $\rho=0 \mathrm{~m}, z=10 \mathrm{~km}$ and (b) $\rho=5 \mathrm{~m}, z=10 \mathrm{~km}$ for different values of $C_{n}{ }^{2}$ and source conditions are given in Fig. 5 and the corresponding values of the relative spectral shift are compiled in Table 1 . The other calculation parameters are the same as those in Fig. 1. It is interesting to find that the evolution of on-axis normalized spectral intensity $S^{\prime}(\rho, z, \omega)$ for the three source 


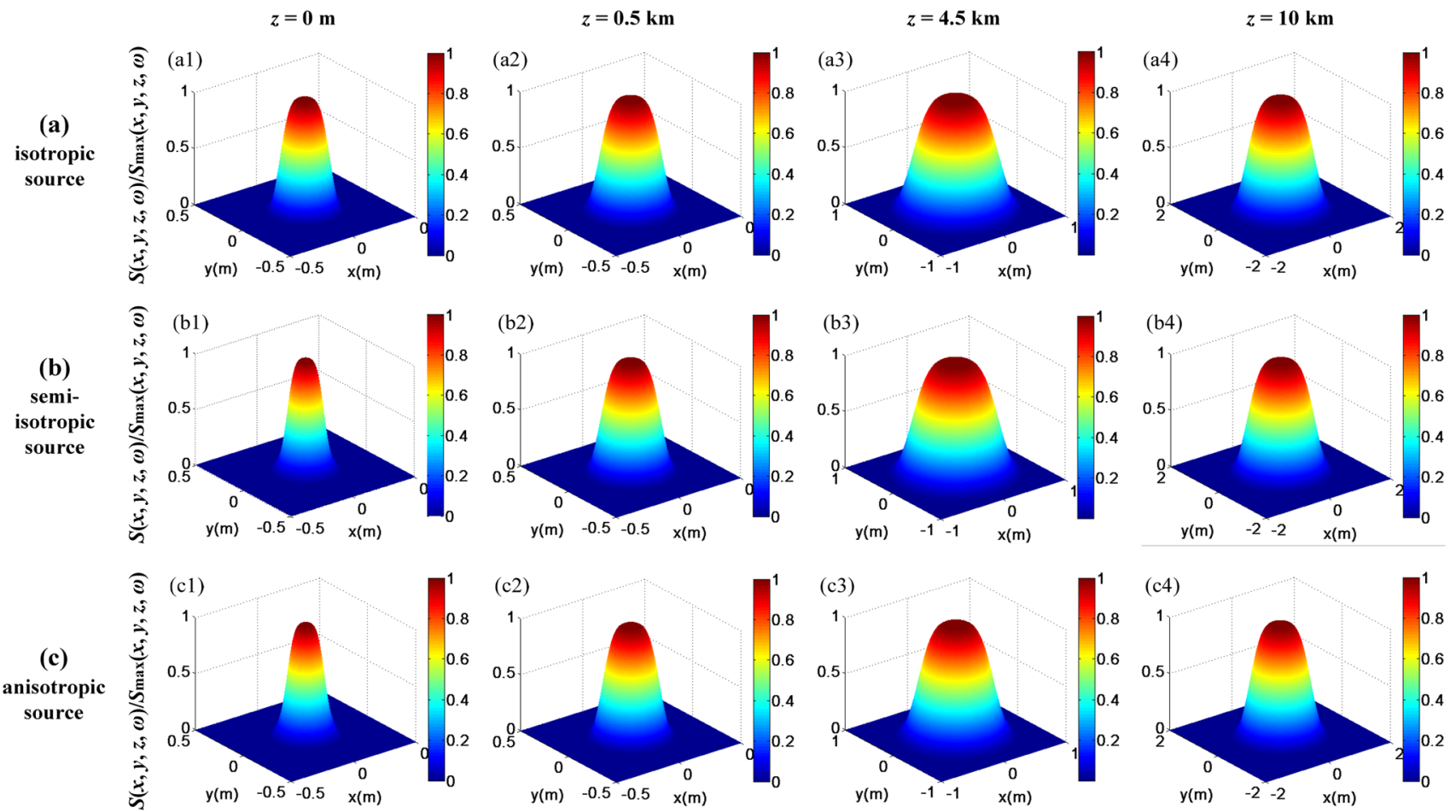

Fig. 3 Normalized 3D-spectral intensity distribution $S(x, y, z$, $\omega) / S_{\max }(x, y, z, \omega)$ of a partially polarized PEGSM beam for different source conditions a isotropic source, b semi-isotropic source, and c anisotropic source at several propagation distances in a horizontal homogeneous and isotropic turbulent atmosphere

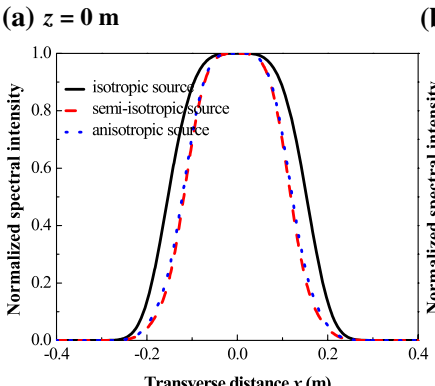

(b) $z=0.5 \mathrm{~km}$

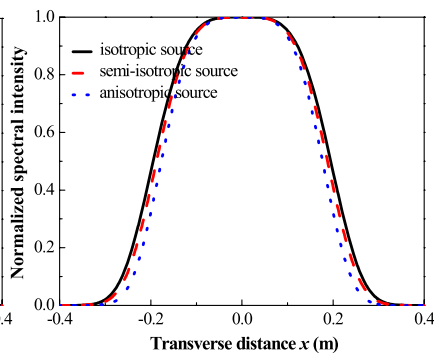

Fig. 4 Cross line $(y=0)$ of the normalized intensity distribution $S(x$, $0, z, \omega) / S_{\max }(x, 0, z, \omega)$ of a partially polarized PEGSM beam at several propagation distances a $z=0 \mathrm{~m}, \mathbf{b} z=0.5 \mathrm{~km}, \mathbf{c} z=4.5 \mathrm{~km}$, and $\mathbf{d}$

conditions almost coincide. In comparison of Fig. 5a1-a3 with Fig. 5b1-b3, we see that the spectrum is blue- and red-shifted in comparison with the original spectrum for $\rho=0 \mathrm{~m}$ and $\rho=5 \mathrm{~m}$, respectively and the value of the blue shift (or red shift) decreases with the increase in $C_{n}^{2}$. Taking the isotropic source as an example, for $\rho=0 \mathrm{~m}$, the normalized spectrum shifts in the direction of the larger frequency and the values of the relative spectral shift $\delta \omega / \omega_{0}=0.0007$ $\left(C_{n}{ }^{2}=0\right)$ and $0.0003\left(C_{n}{ }^{2}=10^{-14} \mathrm{~m}^{-2 / 3}\right)$, respectively; for $\rho=5 \mathrm{~m}$, the normalized spectrum shifts in the direction of the smaller frequency and the values of the relative (c) $z=4.5 \mathrm{~km}$

(d) $z=10 \mathrm{~km}$
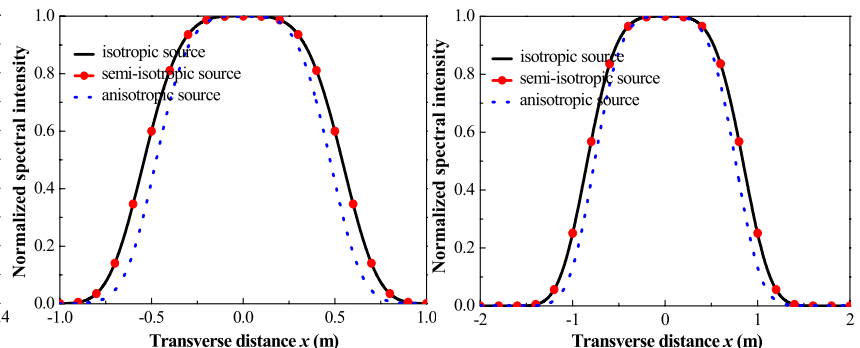

$z=10 \mathrm{~km}$ in a horizontal homogeneous and isotropic turbulent atmosphere for different source conditions

spectral shift $\delta \omega / \omega_{0}=-0.0141\left(C_{n}{ }^{2}=0\right)$ and -0.0067 $\left(C_{n}^{2}=10^{-14} \mathrm{~m}^{-2 / 3}\right)$, respectively. For the case of anisotropic source, values of the red shift are bigger than the other two sources, i.e., the values of the relative spectral shift are $\delta \omega /$ $\omega_{0}=-0.0282\left(C_{n}^{2}=0\right)$ and $-0.0141\left(C_{n}^{2}=10^{-14} \mathrm{~m}^{-2 / 3}\right)$, respectively.

Figure 6 presents the evolution of beam width $W_{x}(z, \omega)$ and $W_{y}(z, \omega)$ of partially polarized PEGSM beams in Eqs. (20) and (21) with the propagation distance $z$ for different values of $C_{n}^{2}$. In the absence of turbulence $\left(C_{n}^{2}=0 \mathrm{~m}^{-2 / 3}\right)$, 
(a1) isotropic source

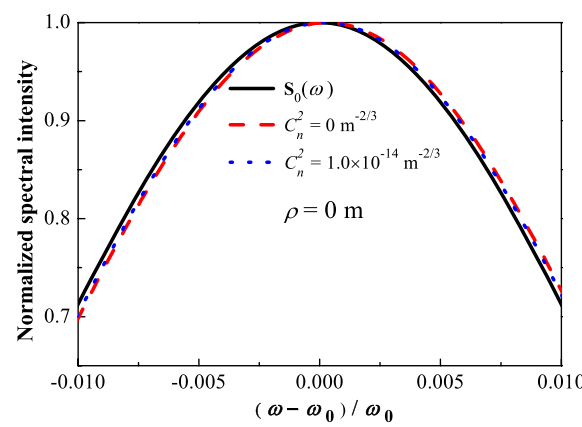

(b1) isotropic source

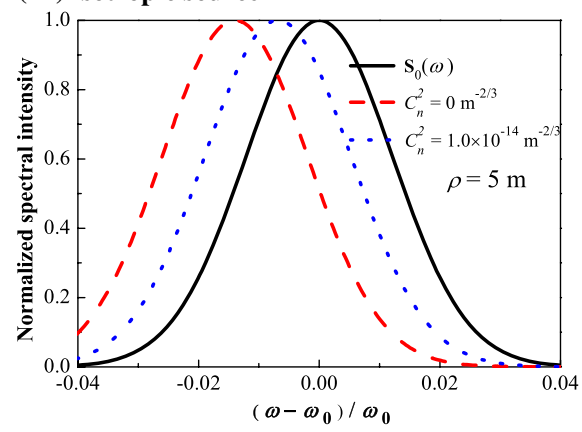

(a2) semi-isotropic source

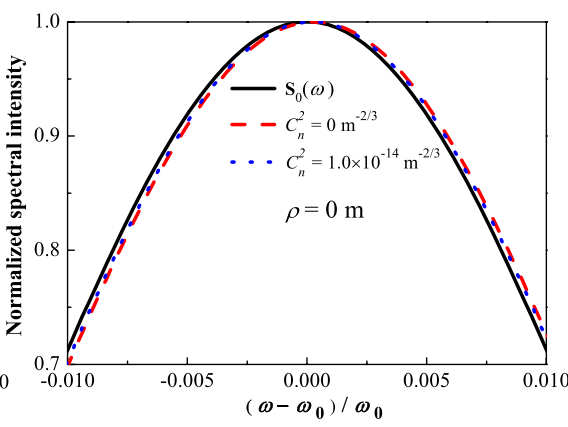

(b2) semi-isotropic source

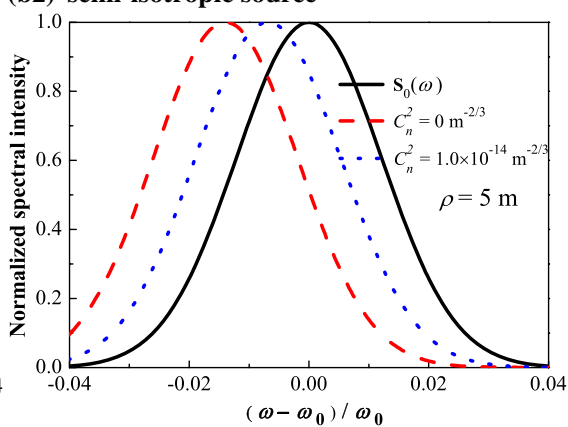

(a3) anisotropic source

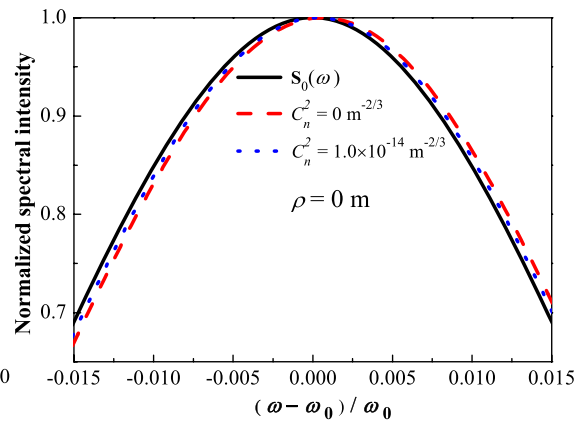

(b3) anisotropic source

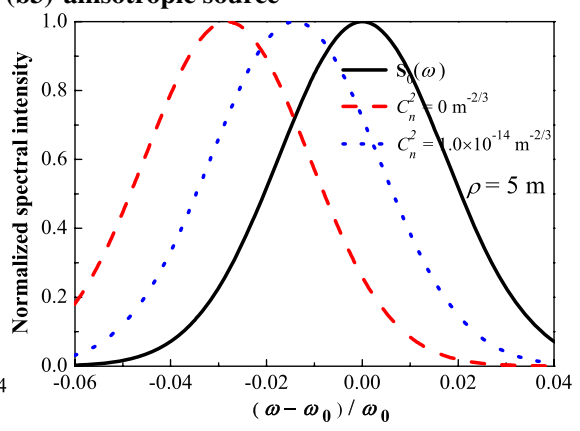

Fig. 5 Evolution of the normalized spectral intensity of partially polarized PEGSM beams as a function of $\left(\omega-/ \omega_{0}\right) / \omega_{0}$ at the obser-

source conditions. The black solid curves denote the original normalvations a $\rho=0 \mathrm{~m}, z=10 \mathrm{~km}$ and $\mathbf{b} \rho=5 \mathrm{~m}, z=10 \mathrm{~km}$ for different

(a) isotropic source

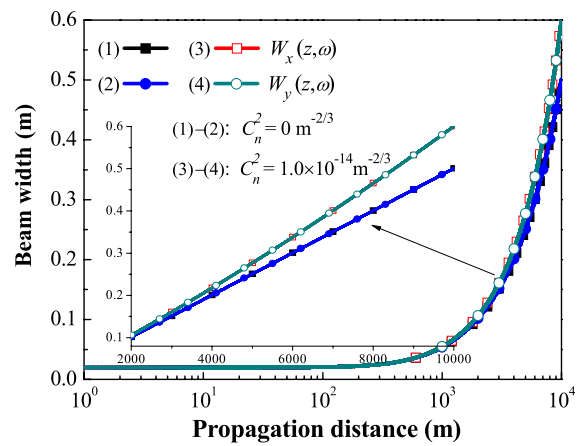

(b) semi-isotropic source

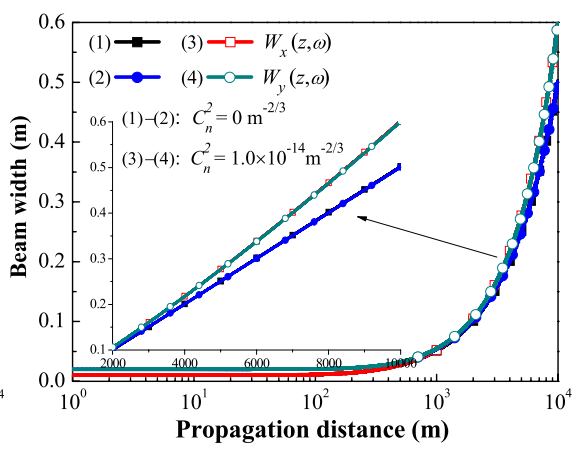

(c) anisotropic source

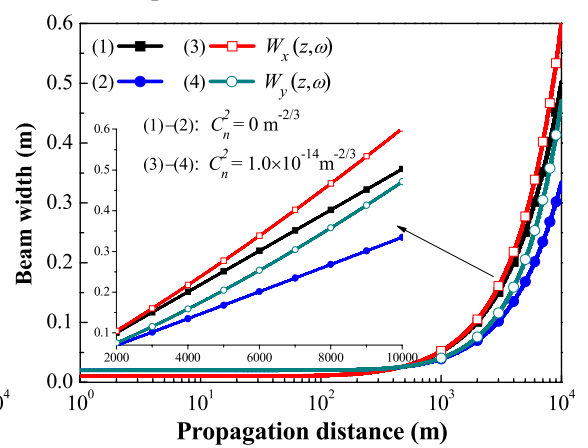

Fig. 6 Changes in the beam width $W_{x}(z, \omega)$ and $W_{y}(z, \omega)$ of partially polarized PEGSM beams versus the propagation distance $z$ with different source conditions a isotropic source, $\mathbf{b}$ semi-isotropic source, and $\mathbf{c}$ anisotropic source for different $C_{n}{ }^{2}$

the fourth term disappears and Eqs. (20) and (21) can be rewritten as:

$W_{i}(z, \omega)=\sigma_{i} \sqrt{1+\frac{c^{2} z^{2}}{4 \omega^{2} \sigma_{i}^{2}}+\frac{c^{2} z^{2}}{\omega^{2} \delta_{i i}^{2}}}, i=x, y$,

where the second term is inverse proportional to the square of $\sigma_{i}$ and the third term is inverse proportional to the square of $\delta_{i i}$. In the presence of turbulence, i.e.
$W_{i}(z, \omega)=\sigma_{i} \sqrt{1+\frac{c^{2} z^{2}}{4 \omega^{2} \sigma_{i}^{2}}+\frac{c^{2} z^{2}}{\omega^{2} \delta_{i i}^{2}}+\frac{1.1 C_{n}^{2} l_{0}^{-1 / 3} z^{3}}{\sigma_{i}^{2}}}, i=x, y$.

As can be seen from Eq. (25), for $z \geq 2000 \mathrm{~m}$, the value of the fourth term is greater than one, i.e., in this case, the turbulence-induced diffraction plays a significant role in the beam width. Comparing Fig. 6 with Table 2, one finds that the beam width $W_{x}(z, \omega)$ and $W_{y}(z, \omega)$ of the former two 
(a) isotropic source

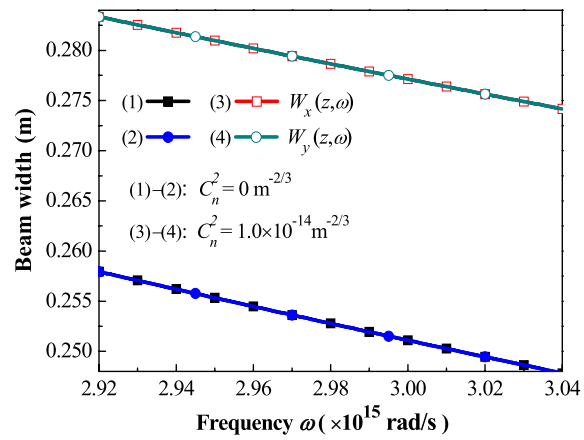

(b) semi-isotropic source

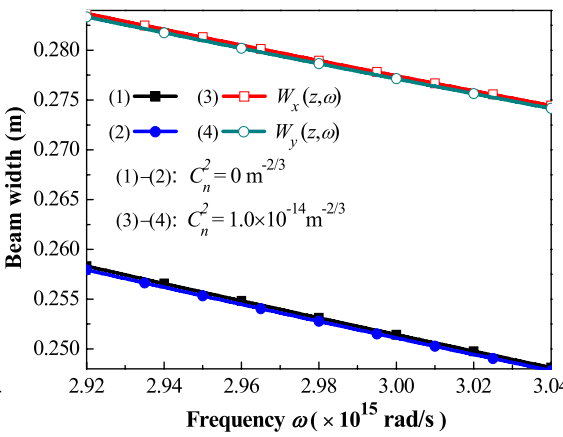

(c) anisotropic source

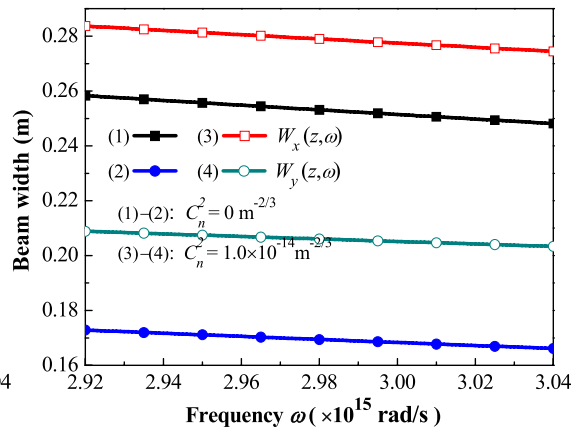

Fig. 7 Changes in the beam width $W_{x}(z, \omega)$ and $W_{y}(z, \omega)$ of partially polarized PEGSM beams versus the frequency $\omega$ with different source conditions a isotropic source, $\mathbf{b}$ semi-isotropic source, and $\mathbf{c}$ anisotropic source for different $C_{n}^{2}$ at $z=5 \mathrm{~km}$

(a) isotropic source

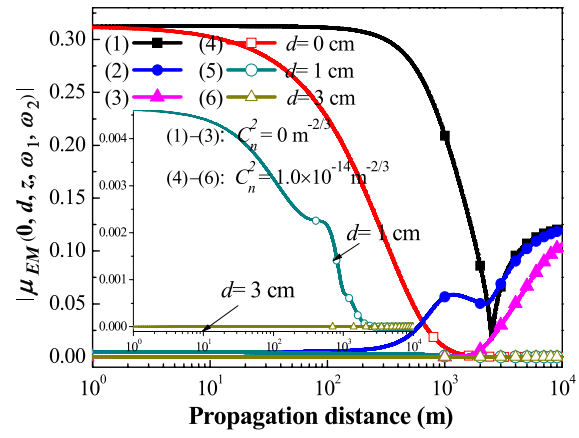

(b) semi-isotropic source

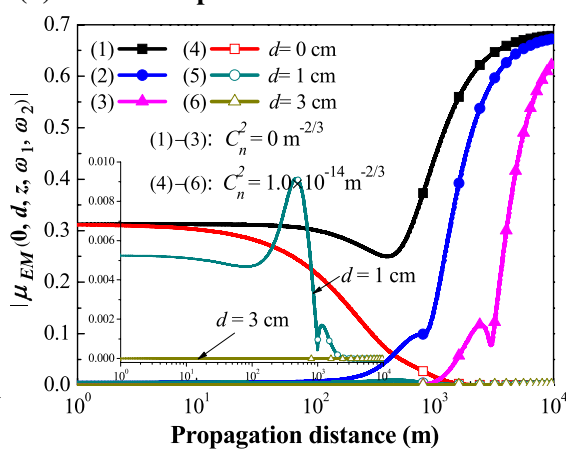

(c) anisotropic source

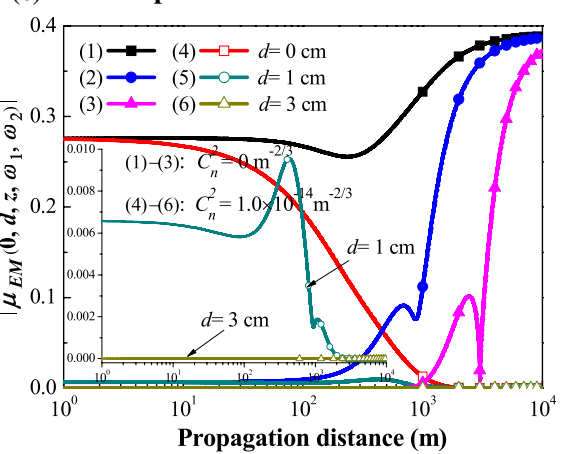

Fig. 8 Changes of the values of the two-frequency, two-point spectral degree of electromagnetic coherence $\left|\mu_{\mathrm{EM}}\left(\boldsymbol{\rho}_{1}, \boldsymbol{\rho}_{2}, z, \omega_{1}, \omega_{2}\right)\right|$ versus propagation distance $z$ with different refractive index structure con- stant $C_{n}^{2}$ and transverse distance $\left(\boldsymbol{\rho}_{1}=0, \boldsymbol{\rho}_{2}=d\right)$ under three different source conditions, where a isotropic source, b semi-isotropic source, and $\mathbf{c}$ anisotropic source source conditions are almost the same. This implies that the partially polarized PEGSM beams with the two source conditions exhibit the same beam profile in the far field region. Moreover, the beam spreads more seriously in the presence of turbulence. On the other hand, the source condition has a great influence on the beam size (see Fig. 6c; also see Table 2). For the case of $0 \mathrm{~m} \leq z \leq 1000 \mathrm{~m}$, the turbulenceinduced diffraction is smaller than the initial beam parameters-induced diffraction, which leads to $W_{x}(z, \omega)<W_{y}(z, \omega)$ within this range. For $z>2000 \mathrm{~m}$, the beam width in the $y$ direction is smaller than that in the $x$ direction. This implies that both the source parameters and turbulence-induced diffraction impact on the beam width.

Figure 7 examines the evolution of beam width $W_{x}(z$, $\omega)$ and $W_{y}(z, \omega)$ of partially polarized PEGSM beams as a function of the frequency $\omega$ for different $C_{n}{ }^{2}$ at $z=5 \mathrm{~km}$. One finds that the variation of the beam size with frequency is not obvious and the beam width in the presence of turbulence is larger than that in the absence of turbulence. This indicates that turbulence-induced diffraction has a larger effect on the beam size and the magnitude of the frequency hardly affects it. Furthermore, it is clear that the beam width $W_{x}(z, \omega)$ and $W_{y}(z, \omega)$ in the anisotropic source cases are different from that two cases. i.e., the beam width difference in the $x$ and $y$ directions is greater.

Now, let us examine the evolution of the two-frequency, two-point spectral degree of electromagnetic coherence $\mu_{\mathrm{EM}}$ $\left(0, d, z, \omega_{1}, \omega_{2}\right)$ in turbulent atmosphere. We employ Eq. (23) to compute $\mu_{\mathrm{EM}}\left(0, d, z, \omega_{1}, \omega_{2}\right)$. The results are plotted in Figs. 8 and 9. Figure 8 presents the evolution of $\mu_{\mathrm{EM}}(0, d$, $\left.z, \omega_{1}, \omega_{2}\right)$ as a function of propagation distance $z$ with different $C_{n}{ }^{2}$ and transverse distance $d$ for different conditions source parameters. One finds that changes of $\mu_{\mathrm{EM}}(0, d, z$, $\left.\omega_{1}, \omega_{2}\right)$ are similar and different from the former one for the case of latter two sources; under the same $C_{n}{ }^{2},\left|\mu_{\mathrm{EM}}\right|$ of semi-isotropic source is greater than that of anisotropic source ( see Fig. 8 b, c). Meanwhile, we find that $\left|\mu_{\mathrm{EM}}\right|$ varies more rapidly with $z$ as $d$ increases. The values of $\left|\mu_{\mathrm{EM}}\right|$ are kept $\approx 0$ then rise with the increase of $d(d>0)$ in the absence of turbulence; in the presence of turbulence, values of $\left|\mu_{\mathrm{EM}}\right|$ 
(a1) isotropic source

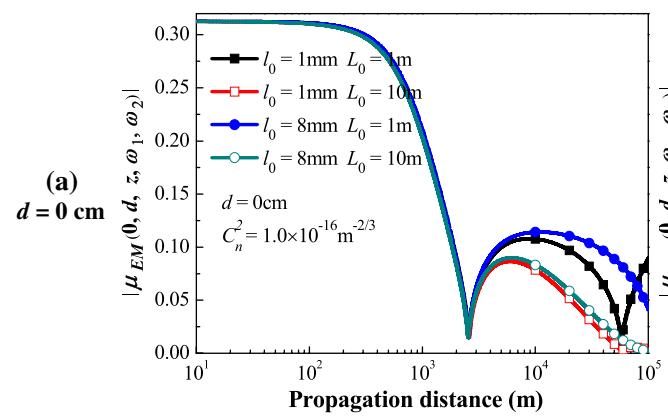

(b1) isotropic source

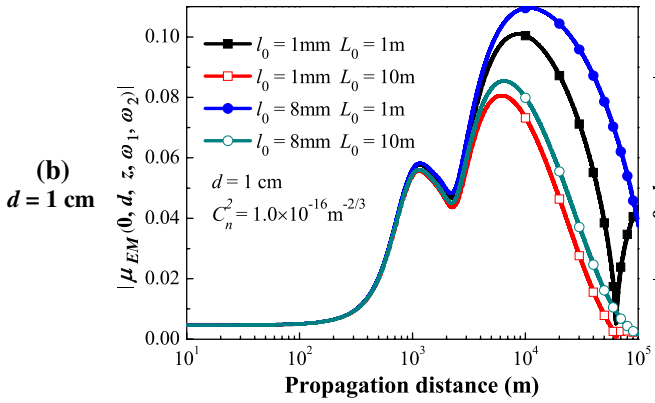

(a2) semi-isotropic source

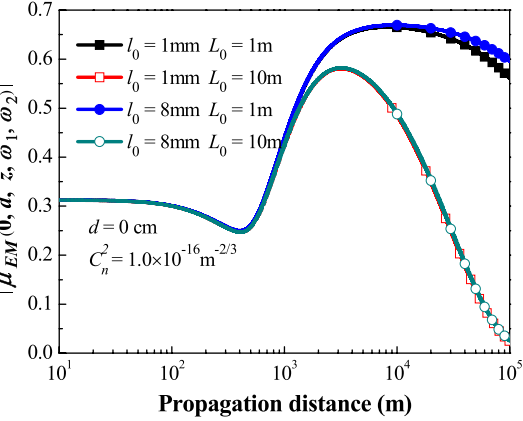

(b2) semi-isotropic source

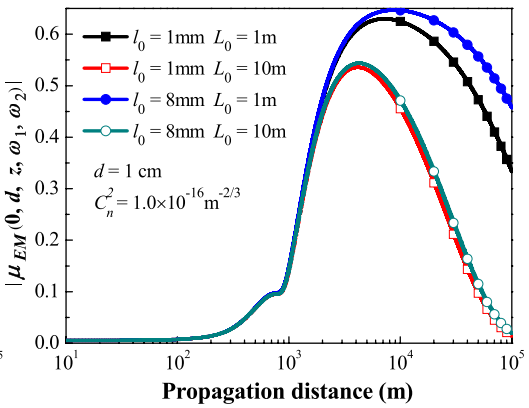

(a3) anisotropic source

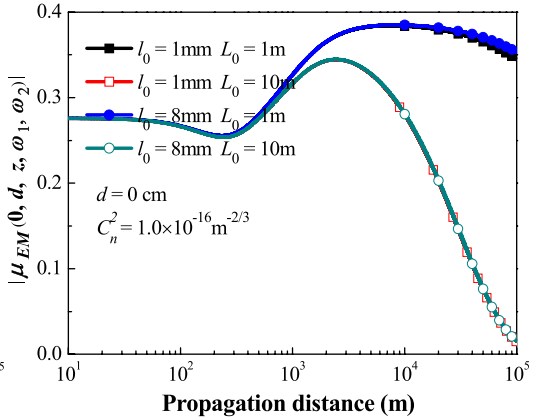

(b3) anisotropic source

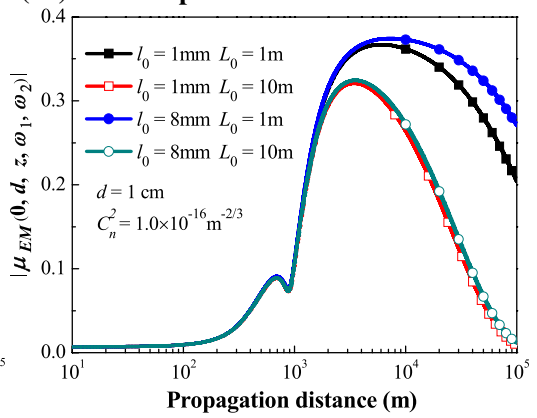

Fig. 9 Changes of the values of the two-frequency, two-point spectral degree of electromagnetic coherence $\left|\mu_{\mathrm{EM}}\left(\boldsymbol{\rho}_{1}, \boldsymbol{\rho}_{2}, z, \omega_{1}, \omega_{2}\right)\right|$ versus propagation distance $z$ with different turbulence inner scale $l_{0}$ and transverse distance $d\left(\rho_{1}=0, \rho_{2}=d\right)$ for different source conditions, where (a1, b1) isotropic source, $(\mathrm{a} 2, \mathrm{~b} 2)$ semi-isotropic source and (a3, b3) anisotropic source (a $d=0 \mathrm{~cm}, \mathbf{b} d=1 \mathrm{~cm}$ )
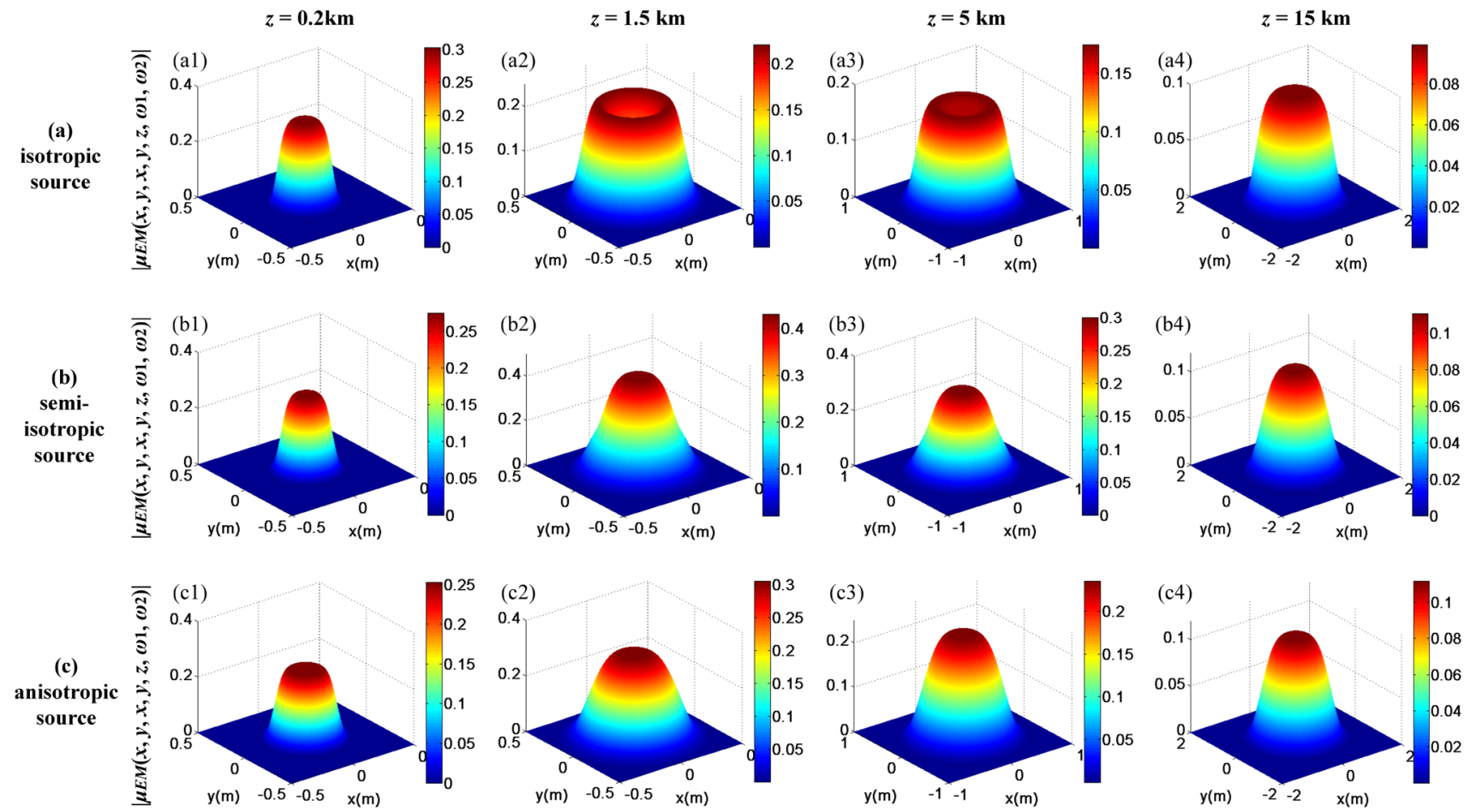

Fig. 10 3D-spectral degree of electromagnetic coherence distribution $\left|\mu_{\mathrm{EM}}\left(x, y, x, y, z, \omega_{1}, \omega_{2}\right)\right|$ of a partially polarized PEGSM beam for different source conditions a isotropic source, b semi-isotropic source, and $\mathbf{c}$ anisotropic source at several propagation distance in a horizontal homogeneous and isotropic turbulent atmosphere 
(a) $z=0.2 \mathrm{~km}$
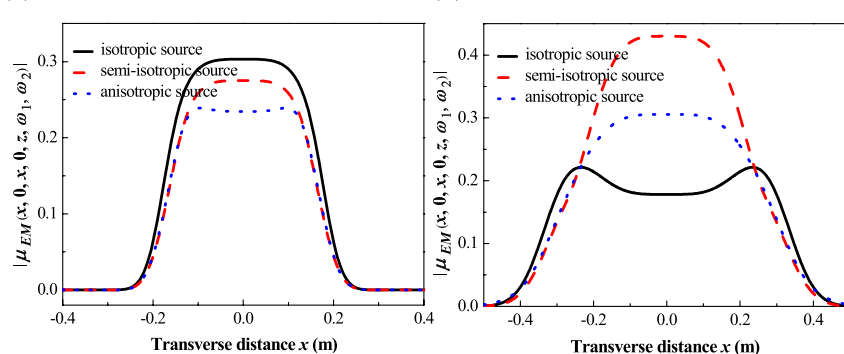

Fig. 11 Cross line $(y=0)$ of the spectral degree of electromagnetic coherence distribution $\left|\mu_{\mathrm{EM}}\left(x, 0, x, 0, z, \omega_{1}, \omega_{2}\right)\right|$ of a partially polarized PEGSM beam at several propagation distances a $z=0.2 \mathrm{~km}$, b (b) $z=1.5 \mathrm{~km}$ (c) $z=5 \mathrm{~km}$

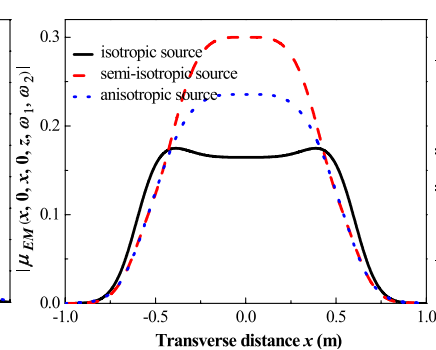

(d) $z=15 \mathrm{~km}$

$z=1.5 \mathrm{~km}, \mathbf{c} z=5 \mathrm{~km}$, and $\mathbf{d} z=15 \mathrm{~km}$ in a horizontal homogeneous and isotropic turbulent atmosphere

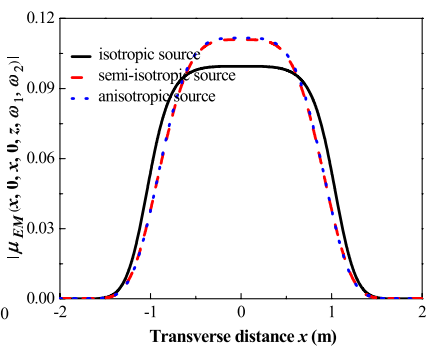

$5 \mathrm{~km}$ in a horizontal homogeneous
Table 1 The values of relative spectral shift corresponding to Fig. 5

\begin{tabular}{|c|c|c|c|c|c|c|c|}
\hline \multicolumn{2}{|c|}{$\delta \omega / \omega_{0}=\left(\omega_{\max }-/ \omega_{0}\right) / \omega_{0}$} & \multicolumn{3}{|c|}{ (a) $\rho=0 \mathrm{~m}$} & \multicolumn{3}{|c|}{ (b) $\rho=5 \mathrm{~m}$} \\
\hline Source conditions & & (a1) & (a2) & (a3) & (b1) & (b2) & (b3) \\
\hline$S_{0}$ & & 0 & 0 & 0 & 0 & 0 & 0 \\
\hline \multirow[t]{2}{*}{$C_{n}^{2} / \mathrm{m}^{-2 / 3}$} & 0 & 0.0007 & 0.0007 & 0.0007 & -0.0141 & -0.0141 & -0.0282 \\
\hline & $1.0 \times 10^{-14}$ & 0.0003 & 0.0003 & 0.0003 & -0.0067 & -0.0067 & -0.0141 \\
\hline
\end{tabular}

\begin{tabular}{|c|c|c|c|c|c|c|c|c|c|}
\hline \multirow[t]{2}{*}{$C_{n}^{2} / \mathrm{m}^{-2 / 3}$} & \multicolumn{3}{|c|}{$z=0.5 \mathrm{~km}$} & \multicolumn{3}{|l|}{$z=5 \mathrm{~km}$} & \multicolumn{3}{|l|}{$z=10 \mathrm{~km}$} \\
\hline & (a) & (b) & (c) & (a) & (b) & (c) & (a) & (b) & (c) \\
\hline \multicolumn{10}{|l|}{$W_{x}$} \\
\hline 0 & 0.03204 & 0.02704 & 0.02704 & 0.25111 & 0.25145 & 0.25145 & 0.50102 & 0.50259 & 0.50259 \\
\hline $1.0 \times 10^{-14}$ & 0.03225 & 0.02729 & 0.02729 & 0.27714 & 0.27744 & 0.27744 & 0.60085 & 0.60216 & 0.60216 \\
\hline \multicolumn{10}{|l|}{$W_{y}$} \\
\hline 0 & 0.03204 & 0.03204 & 0.02606 & 0.25111 & 0.25111 & 0.26832 & 0.50102 & 0.50102 & 0.33487 \\
\hline $1.0 \times 10^{-14}$ & 0.03225 & 0.03225 & 0.02633 & 0.27714 & 0.27714 & 0.20514 & 0.60085 & 0.60085 & 0.47131 \\
\hline
\end{tabular}

Table 2 The special spectral parameters corresponding to Fig. 6 are kept smaller than 0.01 with the increase of $d(d>0)$. For $d=0$, i.e., two-frequency, one-point spectral degree of electromagnetic coherence $\mu_{\mathrm{EM}}\left(0,0, z, \omega_{1}, \omega_{2}\right)$, it can be seen from Fig. 8a that, for $0 \mathrm{~m} \leq z \leq 100 \mathrm{~m}$, the value of $\left|\mu_{\mathrm{EM}}\right|$ remains constant in the absence of turbulence $\left(C_{n}^{2}=0\right)$, then falls rapidly to 0 until $z \approx 3000 \mathrm{~m}$, finally increases quickly. On the other hand, in the presence of turbulence, the value of $\left|\mu_{\mathrm{EM}}\right|$ decreases slightly over a short distance, then drops sharply to 0 . A comparison of Fig. $8 \mathrm{~b}$ with Fig. 8c, in the absence of turbulence, with the increase of $z$, the value of $\left|\mu_{\mathrm{EM}}\right|$ almost stays unchanged, secondly gradually decreases, then increases rapidly and eventually remains constant; in the presence of turbulence, the evolution of $\left|\mu_{\mathrm{EM}}\right|$ agree well with the isotropic source case and the value of $\left|\mu_{\mathrm{EM}}\right|$ in the semi-istropic case is the biggest. Therefore, it can be concluded that the source parameters play a great role in the spectral degree of electromagnetic coherence.

Figure 9 demonstrates the evolution of $\mu_{\mathrm{EM}}\left(0, d, z, \omega_{1}\right.$, $\omega_{2}$ ) versus $z$ for four kinds of turbulence scale and transverse distance $d$ (Fig. 9a: $d=0 \mathrm{~cm}, \mathrm{~b}: d=1 \mathrm{~cm}$ ) under three different source conditions. As seen in Fig. 9a and recalling Fig. $8 \mathrm{a}-\mathrm{c}$, there is a slight change in $\left|\mu_{\mathrm{EM}}\right|$ for $z \leq 100 \mathrm{~m}$. This indicates that the turbulence scale has little effect on the $\left|\mu_{\mathrm{EM}}\right|$ over the distance range. One finds from Fig. 9a1 and b1 that, for the case of $l_{0}=1 \mathrm{~mm}$ and $L_{0}=1 \mathrm{~m}$, when $z>100 \mathrm{~km}$, the evolution of $\left|\mu_{\mathrm{EM}}\right|$ appears abnormal compared with the other three cases. Therefore, it can be concluded that for $5 \mathrm{~km} \leq z \leq 50 \mathrm{~km}$, and $L_{0}$ fixed, the bigger the $l_{0}$ is, the larger the $\left|\mu_{\mathrm{EM}}\right|$ becomes. For $l_{0}$ fixed, the smaller the $L_{0}$ is, the larger the $\left|\mu_{\mathrm{EM}}\right|$ becomes; and the smaller $l_{0}$ is, the bigger the difference becomes.

Figure 9b1-b3 illustrate that, the evolution of $\mu_{\mathrm{EM}}(0, d, z$, $\omega_{1}, \omega_{2}$ ) versus $z$ for four kinds of turbulence scale is similar to the corresponding former three figures (see Fig. 9a1-a3). To draw a conclusion that turbulence inner scale and outer scale play a great role in $\left|\mu_{\mathrm{EM}}\right|$ in the far field region. Moreover, the source conditions have an important effect on $\left|\mu_{\mathrm{EM}}\right|$. Under the same conditions, the $\left|\mu_{\mathrm{EM}}\right|$ of semi-isotropic source is higher than the other two sources. 
Finally, to learn more about the coherence property, by applying Eq. (22) and choosing $\boldsymbol{\rho}_{1}=\boldsymbol{\rho}_{2}=(x, y)$, we calculate in Fig. 10 the 3D-spectral degree of electromagnetic coherence distribution $\left|\mu_{\mathrm{EM}}\left(x, y, x, y, z, \omega_{1}, \omega_{2}\right)\right|$ of a partially polarized PEGSM beam at several propagation distances in the turbulent atmosphere for different source conditions. Meanwhile, we also calculate in Fig. 11 the cross line $(y=0)$ of the spectral degree of electromagnetic coherence distribution $\mid \mu_{\mathrm{EM}}(x, 0, x$, $\left.0, z, \omega_{1}, \omega_{2}\right)$ l of a partially polarized PEGSM beam at several propagation distances for different source conditions. One sees from Figs. 10 and 11 that, for the case of semi-isotropic source and anisotropic source, the spectral degree of electromagnetic coherence exhibits flat-topped Gaussian distribution with circular symmetry, and both the shape and the symmetry keeps unchanged during transmission; the value of $\left|\mu_{\mathrm{EM}}\right|$ increases and then gradually decreases with the increase of $z$. For the case of isotropic source, the shape of the spectral degree of electromagnetic coherence changes during transmission: it exhibits concave distribution at a few kilometers distance; the value of $\left|\mu_{\mathrm{EM}}\right|$ gradually decreases with the increase of propagation distance. It can be concluded that the source parameters play a crucial role in determining the correlation property.

\section{Conclusion}

In summary, the spectral and coherent properties of an isotropic, semi-isotropic, and anisotropic partially polarized pulsed electromagnetic beams propagation upon a horizontal homogeneous and isotropic atmospheric turbulence channel have been examined. With the help of numerical calculations, we have studied the changes in the beam width, spectral, and coherent properties for the beams mentioned. It is shown that, both the source parameters and turbulent atmosphere impact on the spectrum distribution, beam width, and spectral degree of electromagnetic coherence distribution of the partially polarized PEGSM beams. Within a short distance range, source parameters induced diffraction plays a major role; after further propagation, the turbulence-induced diffraction plays a dominant role. It is also found that after passing through a turbulent atmosphere, the spectrum at the far zone shifts towards red shift or blue shift, compared with the source spectrum. In particular, the findings have shown that there is a considerable difference in evolution of the three kinds of partially polarized pulsed electromagnetic beams during propagation. To draw a conclusion, source parameters have little effect on the normalized spectral distribution; the beam width in the case of anisotropic source is smaller than that of the other two sources; and the source parameters play a crucial role in determining the correlation property.

The research outlined in this work provides a better understanding of the second-order statistics of a partially polarized PEGSM beam propagating through atmospheric turbulence in the space frequency domain. Some theoretical results in this paper will be widely applied in the free-space optical communication, information encoding and exchange, and lidar system.

Acknowledgement This work is financed by the National Natural Science Foundation of China (11504286). The program is supported by the International Technology Collaborative Center for Advanced Optical Manufacturing and Optoelectronic Measurement, and the Shaanxi Key Laboratory of Photoelectric Measurement and Instrument Technology.

Open Access This article is licensed under a Creative Commons Attribution 4.0 International License, which permits use, sharing, adaptation, distribution and reproduction in any medium or format, as long as you give appropriate credit to the original author(s) and the source, provide a link to the Creative Commons licence, and indicate if changes were made. The images or other third party material in this article are included in the article's Creative Commons licence, unless indicated otherwise in a credit line to the material. If material is not included in the article's Creative Commons licence and your intended use is not permitted by statutory regulation or exceeds the permitted use, you will need to obtain permission directly from the copyright holder. To view a copy of this licence, visit http://creativecommons.org/licenses/by/4.0/.

\section{References}

1. Y.H. Yang, M. Mandehgar, D.R. Grischkowsky, Broadband THz pulse transmission through the atmosphere. IEEE Trans. Thz. Sci. Technol. 1(1), 264-273 (2011)

2. H.M. Masoudi, M.S. Akond, Time-domain BPM technique for modeling ultrashort pulse propagation in dispersive optical structures: analysis and assessment. J. Lightwave Technol. 32(10), 1936-1943 (2014)

3. M. Gebhardt, C. Gaida, F. Stutzki, S. Hadrich, C. Jauregui, J. Limpert, A. Tunnermann, Impact of atmospheric molecular absorption on the temporal and spatial evolution of ultra-short optical pulses. Opt. Express 23(11), 13776-13787 (2015)

4. R. Dutta, A.T. Friberg, G. Genty, J. Turunen, Two-time coherence of pulse trains and the integrated degree of temporal coherence. J. Opt. Soc. Am. A 32(9), 1631-1637 (2015)

5. P. Huang, S.B. Fang, H.D. Huang, X. Hou, Z.Y. Wei, Coherent synthesis of ultrashort pulses based on balanced optical cross- correlator. Acta Phys. Sin. 67(24), 244204 (2018)

6. J. Papeer, I. Dey, M. Botton, Z. Henis, A.D. Lad, M. Shaikh, D. Sarkar, K. Jana, S. Tata, S.L. Roy, Y.M. Ved, G.R. Kumar, A. Zigler, Towards remote lightning manipulation by meters-long plasma channels generated by ultra-short-pulse high-intensity lasers. Sci. Rep. 9(1), 407 (2019)

7. P. Vahimaa, J. Turunen, Independent-elementary-pulse representation for non-stationary fields. Opt. Express 14(12), 5007-5012 (2006)

8. M. Gao, Y. Li, H. Lv, L. Gong, Polarization properties of polarized and partially coherent electromagnetic Gaussian-Schell model pulse beams on slant path in turbulent atmosphere. Infrared Phys. Technol. 67, 98-106 (2014)

9. P. Paakkonen, J. Turunen, P. Vahimaa, A.T. Friberg, F. Wyrowski, Partially coherent Gaussian pulses. Opt. Commun. 204(1-6), 53-58 (2002)

10. Q. Lin, L.G. Wang, S.Y. Zhu, Partially coherent light pulse and its propagation. Opt. Commun. 219, 65-70 (2003) 
11. J. Lancis, V. Torres-Company, E. Silvestre, P. Andre, Space-time analogy for partially coherent plane-wave-type pulses. Opt. Lett. 30(22), 2973-2975 (2005)

12. V. Torres-Company, G. Minguez-Vega, J. Lancis, A.T. Friberg, Controllable generation of partially coherent light pulses with direct space-to-time pulse shaper. Opt. Lett. 32(12), 1608-1610 (2007)

13. H. Lajunen, J. Tervo, P. Vahimaa, Overall coherence and coherent-mode expansion of spectrally partially coherent plane-wave pulses. J. Opt. Soc. Am. A 21, 2117-2123 (2004)

14. H. Lajunen, P. Vahimaa, J. Tervo, Theory of spatially and spectrally partially coherent pulses. J. Opt. Soc. Am. A 22(8), 15361545 (2005)

15. L.G. Wang, N.H. Liu, Q. Lin, S.Y. Zhu, Propagation of coherent and partially coherent pulses through one-dimensional photonic crystals. Phys. Rev. E 70(1), 016601 (2004)

16. H. Lajunen, J. Turunen, P. Vahimaa, J. Tervo, F. Wyrowski, Spectrally partially coherent pulse trains in dispersive media. Opt. Commun. 255(1-3), 12-22 (2005)

17. H. Lajunen, V. Torres-Company, J. Lancis, E. Silvestre, P. Andres, Pulse-by-pulse method to characterize partially coherent pulse propagation in instantaneous nonlinear media. Opt. Express 18(14), 14979-14991 (2010)

18. D.J. Liu, X.X. Luo, G.Q. Wang, Y.C. Wang, Spectral and coherence properties of spectrally partially coherent Gaussian Schellmodel pulsed beams propagating in turbulent atmosphere. Curr. Opt. Photonics 1(4), 271-277 (2017)

19. Y.Q. Li, Z.S. Wu, M.J. Wang, Partially coherent Gaussian-Schell model pulse beam propagation in slant atmospheric turbulence. Chin. Phys. B 23(6), 064216 (2014)

20. C.Y. Chen, H.M. Yang, Y. Lou, S.F. Tong, Second-order statistics of Gaussian Schell-model pulsed beams propagating through atmospheric turbulence. Opt. Express 19(16), 15196-15204 (2011)

21. A.T. Friberg, T. Setala, Electromagnetic theory of optical coherence [invited]. J. Opt. Soc. Am. A 33(12), 2431-2442 (2016)

22. E. Wolf, Unified theory of coherence and polarization of random electromagnetic beams. Phys. Lett. A 312, 263-267 (2003)

23. H. Roychowdhury, G.P. Agrawal, E. Wolf, Changes in the spectrum, in the spectral degree of polarization, and in the spectral degree of coherence of a partially coherent beam propagating through a gradient-index fiber. J. Opt. Soc. Am. A 23(4), 940-948 (2006)

24. X.Y. Du, D.M. Zhao, Changes in the polarization and coherence of a random electromagnetic beam propagating through a misaligned optical system. J. Opt. Soc. Am. A 25(3), 773-779 (2008)
25. S.G. Hanson, W. Wang, M.L. Jakobsen, M. Takeda, Coherence and polarization of electromagnetic beams modulated by random phase screens and their changes through complex ABCD optical systems. J. Opt. Soc. Am. A 25(9), 2338-2346 (2008)

26. C.L. Ding, L.Z. Pan, B.D. Lü, Changes in the spectral degree of polarization of stochastic spatially and spectrally partially coherent electromagnetic pulses in dispersive media. J. Opt. Soc. Am. B 26(9), 1728-1735 (2009)

27. C.L. Ding, L.Z. Pan, B.D. Lü, Characterization of stochastic spatially and spectrally partially coherent electromagnetic pulsed beams. New J. Phys. 11, 083001 (2009)

28. C.L. Ding, Z.G. Zhao, L.Z. Pan, B.D. Lü, Generalized Stokes parameters of stochastic spatially and spectrally partially coherent electromagnetic pulsed beams. Opt. Commun. 283(22), 4470 $4477(2010)$

29. C.L. Ding, Z.G. Zhao, L.Z. Pan, X. Yuan, B.D. Lü, Propagation of the degree of cross-polarization of stochastic electromagnetic pulsed beams in free space. Opt. Laser Technol. 43(7), 1179-1183 (2011)

30. C.L. Ding, Z.G. Zhao, X.F. Li, L.Z. Pan, X. Yuan, Influence of turbulent atmosphere on polarization properties of stochastic electromagnetic pulsed beams. Chin. Phys. Lett. 28(2), 024214 (2011)

31. D.J. Liu, Y.C. Wang, G.Q. Wang, H.M. Yin, J.R. Wang, The influence of oceanic turbulence on the spectral properties of chirped Gaussian pulsed beam. Opt. Laser Technol. 82, 76-81 (2016)

32. T. Voipio, T. Setala, A.T. Friberg, Partial polarization theory of pulsed optical beams. J. Opt. Soc. Am. A 30(1), 71-81 (2013)

33. O. Korotkova, T.D. Visser, E. Wolf, Polarization properties of stochastic electromagnetic beams. Opt. Commun. 281, 515-520 (2008)

34. C.L. Ding, Y.J. Cai, Y.T. Zhang, L.Z. Pan, Scattering-induced changes in the degree of polarization of a stochastic electromagnetic plane-wave pulse. J. Opt. Soc. Am. A 29(6), 1078-1090 (2012)

35. C.Y. Young, Broadening of ultrashort optical pulses in moderate to strong turbulence. Proc. SPIE 4821, 74-81 (2002)

36. I. Sreenivasiah, A. Ishimaru, Beam wave two-frequency mutualcoherence function and pulse propagation in random media: an analytic solution. Appl. Opt. 18(10), 1613-1618 (1979)

37. B. Ghafary, M. Alavinejad, Changes in the state of polarization of partially coherent flat-topped beam in turbulent atmosphere for different source conditions. Appl. Phys. B Lasers Opt. 102(4), 945-952 (2011)

Publisher's Note Springer Nature remains neutral with regard to jurisdictional claims in published maps and institutional affiliations. 